\title{
Prenatal exposure to antipsychotic medication and use of primary health care system in childhood: a population-based cohort study in Denmark
}

This article was published in the following Dove Press journal: Clinical Epidemiology

\author{
Anne Mette Lund Würtz ${ }^{1,2}$ \\ Claus Høstrup Vestergaard' \\ Dorte Rytter ${ }^{2}$ \\ Merete Juul Sørensen ${ }^{3}$ \\ Jakob Christensen ${ }^{4}$ \\ Mogens Vestergaard ${ }^{1,5}$ \\ Bodil Hammer Bech ${ }^{1,2}$ \\ 'Research Unit for General \\ Practice, ${ }^{2}$ Section for Epidemiology, \\ Department of Public Health, Aarhus \\ University, ${ }^{3}$ Regional Centre for \\ Child and Adolescent Psychiatry, \\ ${ }^{4}$ Department of Neurology, Aarhus \\ University Hospital, ${ }^{5}$ Section for \\ General Practice, Department of \\ Public Health, Aarhus University, \\ Aarhus, Denmark
}

Background: Antipsychotic (AP) medication is increasingly used for many health conditions. Prenatal exposure to AP medication has been associated with several adverse outcomes, but the findings remain inconsistent.

Purpose: We aimed to investigate prenatal exposure to AP medication and the use of primary health care system in childhood.

Subjects and methods: All live-born singletons in Denmark during 1997-2012 were identified in the nationwide Danish National Patient Register and followed until December 31, 2013 (n =963,010). Information on prenatal exposure to AP medication was obtained from the Danish Register of Medicinal Product Statistics. Contacts to the general practitioner (GP) were used as a proxy for the overall health of the children. Negative binomial regression was used to calculate incidence rate ratios (IRRs) and 95\% confidence intervals (CIs) for the association between prenatal exposure to AP medication and number and type of GP contacts, excluding routine well-child visits and vaccinations. The models were adjusted for sex and birth date of the child, maternal age, parity, cohabitation status, income, education, smoking status, diagnosis of substance abuse, severe psychiatric disorder, depression and epilepsy as well as the use of antiepileptic drugs, antidepressants, benzodiazepines and insulin.

Results: The prenatally AP-exposed children had 7\% more GP contacts than unexposed children, IRR: 1.07 (95\% CI: 1.03, 1.11). The association was slightly stronger among children of mothers with no diagnosis of severe psychiatric disorder (IRR: $1.08,95 \%$ CI: 1.04-1.13) than among children of mothers with severe psychiatric disorder (IRR: 1.03, 95\% CI: 0.96-1.11), but the difference was not statistically significant. We found no difference between prenatally AP-exposed children and their unexposed siblings, IRR: 1.00 (95\% CI: 0.97-1.04) for total contacts.

Conclusion: Children of women using AP medication in pregnancy had more GP contacts in childhood than children of mothers not using AP medication. However, this might be explained by confounding from maternal behavior and mental illness.

Keywords: antipsychotic medication, prenatal drug exposure, primary health care, general practitioner

\section{Plain language summary}

Correspondence: Bodil Hammer Bech Department of Public Health, Aarhus University, Bartholins Allé 2, DK-8000 Aarhus C, Denmark

Tel +4587167961

Fax +4586124788

Email bhb@ph.au.dk
Why was the study performed? Antipsychotic (AP) medication is used for a number of mental conditions, where many of which require continuous use during pregnancy. However, we do not know if AP medication affects the health of the child. This study investigates whether AP-exposed children receive more services for health problems in general practice compared to unexposed children. 
What did the researchers do and find? This population-based cohort study from Denmark linked information from selected national registers. Exposed children had 7\% more contacts to the general practitioner (GP) during childhood compared to unexposed children, corresponding to a difference of $0.32 \mathrm{GP}$ visits per year. However, no difference in contacts to the GP was found for exposed children compared to their unexposed siblings.

What do these results mean? This study suggests an association between prenatal exposure to AP medication and more contacts to primary health care services in childhood. However, as no difference was found in the sibling analysis the association is more likely because of differences in the underlying health seeking behavior or lifestyle of mothers taking AP during pregnancy compared to mothers not taking this medication than due to the medication itself.

\section{Introduction}

Antipsychotic (AP) medication is used for a number of indications, including psychosis, bipolar disorder and schizophrenia, but it may also be used for disorders such as depression, obsessive-compulsive disorder, pain, anxiety, Tourette's syndrome and autism. ${ }^{1-3}$ The use of AP medication during pregnancy has increased during the past 2 decades, ${ }^{4,5}$ also in Denmark. ${ }^{6}$ As most APs cross the placenta, the consequences for the fetus should be considered. ${ }^{7,8}$ A recent systematic review found that prenatal exposure to secondgeneration AP medication was associated with two times the risk of congenital malformations (odds ratio [OR]: 2.03, 95\% confidence interval [CI]: 1.41-2.93) and 80\% higher risk of preterm birth (OR: $1.85,95 \% \mathrm{CI}: 1.20-2.86) .{ }^{9}$ In the systematic review by Coughlin et al, ${ }^{10}$ no difference between first- and second-generation AP medication in the risk of congenital malformations was found. It was also shown that prenatal exposure to AP medication was associated with more than two times the risk of giving birth to a smallfor-gestational-age child (OR: 2.44, 95\% CI: 1.22-4.86). Yet, these findings remain inconsistent. ${ }^{11}$ Previous studies of prenatally AP-exposed children have mainly focused on the outcomes in newborns and infants, while little is known about the health during childhood. ${ }^{11}$ A few studies, however, have found a short-term delay in development ${ }^{12}$ or neuromotor performance ${ }^{13}$ in children prenatally exposed to AP medication.

Health care services provided by general practitioners (GPs) in Denmark are free of charge, and 98\% of all residents are registered with a specific general practice. The services provided by the GPs are funded by the Danish tax system, and GPs are remunerated through a combination of capitation and fee-for-service. Accordingly, the GPs must carefully register each specific contact and medical procedure performed to obtain payment for the services provided. ${ }^{14}$ The GPs serve as gatekeepers to the rest of the health care system, and all citizens must thus first contact their GP for medical advice concerning all nonemergency symptoms and diseases. ${ }^{15}$ As most diseases are diagnosed and treated in primary care, the services provided by the GPs may be seen as a proxy for the overall health of the citizens.

The aim of the current study was to investigate the association between prenatal exposure to AP medication and use of the primary health care system as measured by the number of GP contacts. If prenatal exposure to AP medication is associated with a higher overall mental or physical morbidity in the offspring, we would expect exposed children to have more GP contacts than unexposed children.

\section{Subjects and methods Study design and population}

The underlying study population has been described previously. ${ }^{16}$ In short, we conducted a population-based cohort study where we included all singleton live-born children in Denmark from January 1, 1997 to December 31, 2012. The children were followed from date of birth until death, emigration or December 31, 2013, whichever came first. The Danish National Patient Register was used to identify all live births by using code DZ38* of the International Classification of Diseases (ICD), 10th revision. ${ }^{17}$ All Danish citizens are assigned a unique personal identification number at birth in the Danish Civil Registration System. ${ }^{18}$ This number was used to link data across registers. In total, 1,019,645 children were born in Denmark in the study period. We excluded 56,635 children for one of the following reasons: they were not singleton $(n=41,713)$, no information was available on their gestational age $(n=5,752)$, they were not registered in the Danish Civil Registration System $(n=5,032)$ or they could not be linked to their mother $(n=4,138)$. This left us with 963,010 eligible children for inclusion in the current study. The study was approved by the Danish Data Protection Agency. All data were anonymized before analysis. Ethical approval was not required according to the Danish regulations due to the register-based nature of the study.

\section{Exposure}

The exposure of interest was the mother's use of AP medication during pregnancy. The Danish Register of Medicinal 
Product Statistics holds records of all prescriptions redeemed since January 1, 1996, ${ }^{19}$ although without information on medical treatment during inpatient hospital admission. From this register, we obtained information on the mother's use of AP medication during pregnancy. AP use was defined as any prescription redeemed with the Anatomical Therapeutic Chemical (ATC) code N05A (antipsychotics) as defined by the World Health Organization. ${ }^{20}$ The first day of the last menstrual period of the mother was estimated by subtracting the gestational age from the birth date of the child. The exposure window was defined as 30 days before the estimated first day of the last menstrual period to the day before birth. Among the 963,010 children included in the study, 2,483 were prenatally exposed to AP medication and 960,527 were not.

\section{Outcome}

The primary outcome of interest was the number and type of contacts to the GP after birth in the period from January 1, 1997 to December 31, 2013. The secondary outcome was the use of specific services provided by the GP for the children in the same period. All contacts to the GPs and the services provided for each patient are electronically registered with specific service codes in the Danish National Health Insurance Service Register. ${ }^{21}$ From this register, we obtained information on the number of consultations in daytime and out-of-hours, the number of telephone and email contacts and the number of diagnostic tests performed during daytime. Contacts related to routine well-child visits and the Danish childhood vaccination program were excluded. In Denmark, three well-child visits are scheduled during a child's first year and then one per year is scheduled up to the age of 5 years. One outcome of the study was the total number of GP contacts per year, including daytime visits, out-of-hour visits, telephone contacts (both daytime and out-of-hours), email contacts (from January 1, 2004) and home visits. Furthermore, we separately studied the number of GP visits in daytime and out-of-hours, telephone contacts and email contacts. We also explored the reasons for the contacts. Among the specific services provided by the GP, we included only activities related to the physical health: blood sample (activity codes 2601 and 2101), B-hemoglobin measurement (activity code 7108), peak flow/spirometry (activity codes 7113, 7121 and 7183), urinary stix (activity code 7101), streptococcal antigen test (activity code 7109) and C-reactive protein (CRP) measurement (activity code 7120). We also investigated positive test results for streptococcal antigen and CRP tests. Information on positive test results was not recorded in the register, but we defined a positive test result as a contact for which an antibiotic prescription was redeemed for the child within 8 days after performance of the streptococcal antigen or CRP test. Included antibiotics were phenoxymethylpenicillin (ATC code J01CE02), amoxicillin (ATC code J01CA04), amoxicillin/clavulanic acid (ATC code J01CR02), clarithromycin (ATC code J01FA09), roxithromycin (ATC code J01FA06) and clindamycin (ATC code J01FF01).

\section{Covariates}

The Danish Civil Registration System was used to obtain information on the sex of the children, maternal age and parity. The Danish National Patient Register was used to obtain information on gestational age, date of birth, Apgar score at 5 min of the children and maternal smoking status at the index pregnancy. ${ }^{17}$ Information on maternal cohabitation status, income and education was obtained from Statistics Denmark. ${ }^{22}$ We identified mothers diagnosed with severe psychiatric disorder (ICD-8 codes 295, 296.1-296.8 and 298.1, and ICD-10 codes F20 and F30-F31) before the end of the index pregnancy through the Danish National Patient Register, ${ }^{17}$ which holds information on all inpatients from 1978 and all outpatients from 1995 onward, and the Danish Psychiatric Central Research Register, which holds information on all inpatients from 1969 and outpatients from 1995 onward.$^{23}$ In the Danish National Patient Register, we identified mothers with a diagnosis of epilepsy (ICD-8 code 345 , and ICD-10 codes G40 and G41). ${ }^{17,24}$ In the Danish Psychiatric Central Research Register, ${ }^{23}$ we identified mothers with a diagnosis of substance abuse (ICD-8 codes 291, 294.3, 303 and 304, and ICD-10 codes F10-F19) or depression (ICD-8 codes 296.0, 298.0 and 300.4, and ICD-10 codes F32-F33). Data on maternal use of antiepileptic drugs (ATC code N03A and N05BA09), antidepressants (ATC code N06A), benzodiazepines (ATC code N05BA and N05CD) and insulin (ATC code A10A) during the exposure window were obtained from the Danish Register of Medicinal Product Statistics.

\section{Data analysis}

The characteristics of the children and their mothers were summarized using mean and standard deviation (SD) or proportions. Incidence rate ratios (IRRs) and corresponding 95\% CIs for each year of follow-up and the entire follow-up period were estimated using a negative binomial regression model applying cluster robust variance to account for correlations in the use of GP services for the children due to multiparity in the mothers. We adjusted the IRRs for sex (male, female), date of birth of the children (date; continuous), maternal age (years; continuous), cohabitation status (married, cohabiting, 
single, missing category), income (quartiles by year and missing category), education ( $<10$ years, $10-15$ years, $>15$ years, missing category), smoking status (nonsmoker, smoker, unknown), diagnosis of substance abuse (yes, no), severe psychiatric disorder (yes, no), depression (yes, no) and epilepsy (yes, no). We also adjusted for use of antiepileptic drugs (yes, no), antidepressants (yes, no), benzodiazepines (yes, no) and insulin (yes, no) at some point during the index pregnancy. Maternal cohabitation status, income, education, substance abuse, depression and severe psychiatric disorders were included as time-varying covariates.

As we only have information on redemption of prescribed AP medication, but no information on whether the drug was taken or not, we made a sensitivity analysis where exposure was defined as two or more redemptions of AP medication during pregnancy.

To explore potential confounding by indication, we stratified the analyses for the primary outcome according to "never" vs "ever" maternal diagnosis of severe psychiatric disorder as registered in the Danish National Patient Register or the Danish Psychiatric Central Research Register before the end of the index pregnancy. In addition, this stratified analysis included only women diagnosed with severe psychiatric disorders in the 5 years prior to the index pregnancy. We further explored potential confounding by indication by comparing the prenatally AP-exposed children with children of mothers who had used APs at some point during the year preceding the index pregnancy but not during the 30 days before the estimated first day of the last menstrual period and during the index pregnancy. In addition, to take into account potential unmeasured confounding from environmental and genetic risk factors and the mother's care-seeking behavior, we studied siblings discordant for prenatal exposure to AP medication and compared the prenatally AP-exposed children with their unexposed siblings in a mixed-model analysis with separate strata for each mother and each child. Furthermore, first- and second-generation AP medications were grouped together but studied separately. Finally, the five most commonly used APs were studied separately.

All statistical analyses were performed using Stata 13 (StataCorp LP, College Station, TX, USA).

\section{Results}

We followed 963,010 children for a median of 9 years (range: 1-16 years). Children prenatally exposed to APs were slightly more likely to be male, born preterm and to have low Apgar score than unexposed children (Table 1). In addition, a higher proportion of exposed children were born in the most recent
Table I Characteristics of the children prenatally exposed and unexposed to AP medication and their mothers at the time of the index pregnancy; mean (SD) or proportion

\begin{tabular}{|c|c|c|}
\hline & $\begin{array}{l}\text { +AP medication } \\
(n=2,483)\end{array}$ & $\begin{array}{l}\text {-AP medication } \\
(n=960,527)\end{array}$ \\
\hline \multicolumn{3}{|l|}{ Characteristics of the children } \\
\hline \multicolumn{3}{|l|}{ Sex, \% } \\
\hline Female & 48 & 49 \\
\hline Male & 52 & 51 \\
\hline \multicolumn{3}{|l|}{ Gestational age } \\
\hline Mean (SD), days & $274(15)$ & $279(13)$ \\
\hline Preterm (<37 weeks), \% & 9 & 5 \\
\hline Term ( $\geq 37$ weeks), $\%$ & 91 & 95 \\
\hline \multicolumn{3}{|l|}{ Birth year, \% } \\
\hline$|997-200|$ & 22 & 32 \\
\hline $2002-2006$ & 28 & 31 \\
\hline $2007-2012$ & 51 & 36 \\
\hline \multicolumn{3}{|l|}{ Apgar score at $5 \mathrm{~min}, \%$} \\
\hline $0-7$ & 3 & I \\
\hline $8-10$ & 97 & 98 \\
\hline Missing value & $<1$ & $<1$ \\
\hline \multicolumn{3}{|l|}{ Characteristics of the mothers } \\
\hline \multicolumn{3}{|l|}{ Maternal age, years } \\
\hline Mean (SD) & $31(6)$ & $30(5)$ \\
\hline$<21, \%$ & 3 & 2 \\
\hline $2 \mathrm{I}-25, \%$ & 17 & 12 \\
\hline $26-30, \%$ & 26 & 34 \\
\hline $31-35, \%$ & 29 & 36 \\
\hline$\geq 36, \%$ & 25 & 17 \\
\hline \multicolumn{3}{|l|}{ Parity, \% } \\
\hline I child & 45 & 44 \\
\hline 2 children & 26 & 37 \\
\hline 3 children & 17 & 14 \\
\hline $4+$ children & 12 & 5 \\
\hline \multicolumn{3}{|l|}{ Cohabitation, \% } \\
\hline Married & 43 & 60 \\
\hline Cohabiting & 31 & 33 \\
\hline Single & 25 & 7 \\
\hline Missing value & $<1$ & $<1$ \\
\hline \multicolumn{3}{|l|}{ Income, \% } \\
\hline Quartile I & 43 & 25 \\
\hline Quartile 2 & 31 & 25 \\
\hline Quartile 3 & 16 & 25 \\
\hline Quartile 4 & 9 & 25 \\
\hline Missing value & $<1$ & $<1$ \\
\hline \multicolumn{3}{|l|}{ Education, \% } \\
\hline$<10$ years & 48 & 18 \\
\hline $10-15$ years & 34 & 45 \\
\hline$>15$ years & 13 & 33 \\
\hline Missing value & 4 & 3 \\
\hline \multicolumn{3}{|l|}{ Smoking status, \% } \\
\hline Nonsmoker & 52 & 80 \\
\hline Smoker & 39 & 15 \\
\hline Unknown & 9 & 5 \\
\hline \multicolumn{3}{|l|}{ Diagnoses, \% } \\
\hline $\begin{array}{l}\text { Severe psychiatric } \\
\text { disorders }\end{array}$ & 27 & $<1$ \\
\hline Substance abuse & 22 & 3 \\
\hline
\end{tabular}

(Continued) 
Table I (Continued)

\begin{tabular}{lll}
\hline & $\begin{array}{l}\text { +AP medication } \\
(\mathbf{n = 2 , 4 8 3 )}\end{array}$ & $\begin{array}{l}\text {-AP medication } \\
(\mathbf{n = 9 6 0 , 5 2 7 )}\end{array}$ \\
\hline Depression & 36 & 2 \\
Epilepsy & 4 & 2 \\
Concurrent drug use, \% & & \\
Antiepileptic drugs & 3 & $<1$ \\
Antidepressants & 49 & 2 \\
Benzodiazepines & 19 & $<1$ \\
Insulin & 1 & 1 \\
\hline
\end{tabular}

Abbreviations: AP, antipsychotic; SD, standard deviation.

years of the study period (2007-2012) compared to unexposed children. Furthermore, the mothers of exposed children were more likely to be older, single, smoker, have short education, have a diagnosis of severe psychiatric disorder, substance abuse, depression or epilepsy, and concurrently take antiepileptic drugs or antidepressants.

The total number of GP contacts during the entire followup period was $46,736,112$. The average unadjusted number of GP contacts among unexposed children was 4.6 contacts per year. On average, children exposed to APs prenatally had 0.32 more contacts per year than unexposed children. In addition, within each year of follow-up, children prenatally exposed to APs had more total contacts, GP visits and phone contacts than unexposed children, although this was not statistically significant within every year (Figure 1). Throughout the entire study period, children prenatally exposed to APs had $7 \%$ more total GP contacts than unexposed children after adjustments for covariates: IRR: 1.07 (95\% CI: 1.03, 1.11; Table 2). Prenatal exposure to AP medication was associated with more GP visits and phone contacts, whereas no association was found with email contacts (Table 2). For the specific services provided at GP contacts, we found prenatal exposure to AP medication to be associated with an $11 \%$ higher risk of having streptococcal antigen (IRR: 1.11, 95\% CI: $1.05,1.18$ ) and 10\% higher risk of CRP (IRR: 1.10, 95\% CI: $1.00,1.21)$ tests performed. We also found a statistically nonsignificant higher risk of having positive test results for these two tests, defined as having redeemed a prescription for antibiotics within 8 days after the test; IRR: 1.07 (95\% CI: $0.98,1.16)$ for positive streptococcal antigen test and IRR: 1.10 (95\% CI: $0.96,1.27)$ for positive CRP test. For blood samples, B-hemoglobin measurements, peak flow/spirometry, and urinary stixs, we found no statistically significant difference between AP-exposed and AP-unexposed children (Table 2). Defining exposure as two or more redemptions of
AP medication during pregnancy did not change the results (data not shown).

When we stratified the analyses according to maternal "never" vs "ever" diagnosis of severe psychiatric disorder, the relative risk estimates were a little stronger among children of mothers with no hospital-based diagnosis of severe psychiatric disorder, except for email contacts (Table 3). Comparing children prenatally exposed to APs $(n=2,483)$ with children of mothers who used APs in the year preceding the pregnancy but not during the pregnancy $(n=3,237)$, we found prenatally AP-exposed children to have slightly fewer GP contacts than children of mothers who used APs at some point before the index pregnancy (Table 4).

Among 2,618 siblings discordant for AP exposure, we found no statistically significant difference in the number of GP contacts between children prenatally exposed to AP medication $(n=1,059)$ and their unexposed siblings $(n=1,559$; Figure 2).

When we grouped APs into first- and second-generation AP medication, we only found an association for firstgeneration AP medication. Children prenatally exposed to first-generation AP medication had 12\% more contacts than unexposed children, IRR: 1.12 (1.08-1.17), while we found no difference in total contacts for children prenatally exposed to second-generation AP medication compared to unexposed children, IRR: 1.00 (0.95-1.05). The most commonly used types of APs were chlorprothixene (N05AF03) (18\%), quetiapine (N05AH04) (17\%), perphenazine (N05AB03) (16\%), olanzapine (N05AH03) (11\%) and zuclopenthixol (N05AF05) (10\%). Children prenatally exposed to chlorprothixene, perphenazine or zuclopenthixol (all first-generation AP medication) had more total GP contacts than children unexposed to any APs, whereas no association was found for quetiapine or olanzapine, which are second-generation AP medication (Figure 3).

\section{Discussion Main findings}

In this large population-based follow-up study, we found prenatal exposure to AP medication to be associated with more GP contacts overall, including GP visits and phone contacts, after adjusting for potential risk factors. However, these associations may be a result of confounding from maternal behavior due to underlying maternal mental illness for two reasons: 1) we found no association when unexposed siblings were used as reference group and 2) the estimates were weak in the strata with maternal diagnosis of severe psychiatric disorder. 

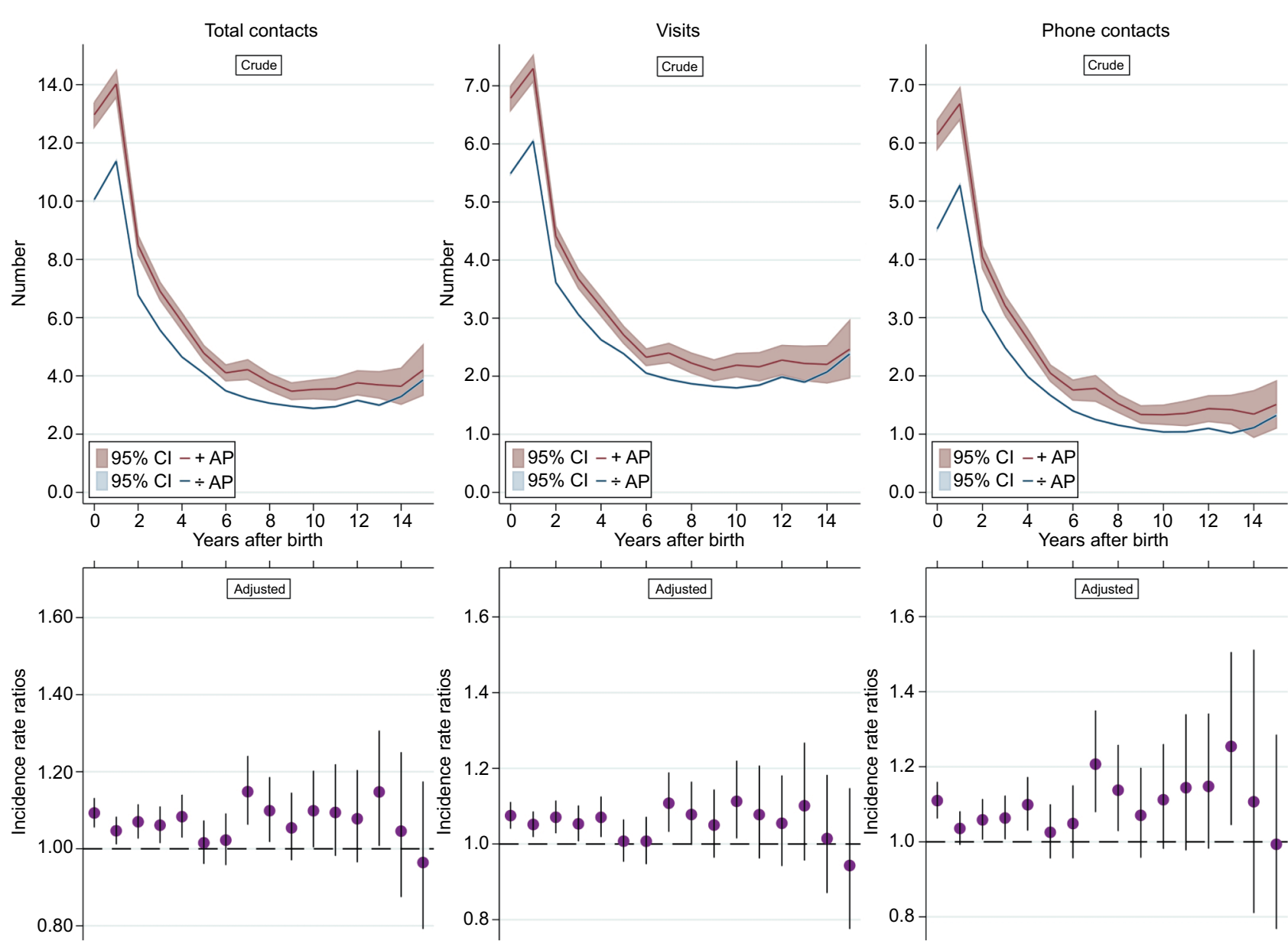

Figure I Type of GP contact by age of the children in I-year intervals comparing prenatally AP-exposed and AP-unexposed children; unadjusted mean number of contacts in top panels and adjusted IRRs $(95 \% \mathrm{Cl})$ in bottom panels.

Notes: IRRs and $95 \% \mathrm{Cls}$ were estimated using negative binomial regression applying cluster robust variance to account for correlations in the children's use of GP services due to multiparity in the mothers. Adjusted for sex and date of birth of the children and for maternal age, parity, cohabitation status, income, education, smoking status, diagnosis of substance abuse, depression and epilepsy as well as use of antiepileptic drugs, antidepressants, benzodiazepines and insulin.

Abbreviations: AP, antipsychotic; $\mathrm{Cl}$, confidence interval; GP, general practitioner; IRR, incidence rate ratio.

Table 2 IRR $(95 \% \mathrm{Cl})$ for type of GP contact and services provided comparing prenatally AP-exposed children to APunexposed children

\begin{tabular}{|c|c|c|}
\hline Contact & $\begin{array}{l}\text { Crude IRR } \\
(95 \% \mathrm{CI}) \\
\end{array}$ & $\begin{array}{l}\text { Adjusted IRR } \\
(95 \% \mathrm{CI})^{\mathrm{a}}\end{array}$ \\
\hline \multicolumn{3}{|l|}{ Type of GP contact } \\
\hline Total contacts & $1.32(1.28-1.36)$ & $\mathrm{I} .07(\mathrm{I} .03-\mathrm{I} . \mathrm{II})$ \\
\hline Visits & $1.27(1.23-1.30)$ & $1.06(1.03-1.09)$ \\
\hline Phone contacts & $1.39(1.34-1.45)$ & $1.08(1.04-1.13)$ \\
\hline Email contacts (from 2004) & $0.82(0.68-0.99)$ & $0.84(0.68-1.0 \mathrm{I})$ \\
\hline \multicolumn{3}{|l|}{ Service at GP contact } \\
\hline Blood sample & I.I $2(0.97-1.29)$ & $1.06(0.92-1.23)$ \\
\hline B-hemoglobin measurement & $1.26(1.10-1.45)$ & $1.04(0.89-1.20)$ \\
\hline Peak flow/spirometry & $1.08(0.86-1.35)$ & $1.14(0.90-1.43)$ \\
\hline Urinary stix & $1.17(1.04-1.32)$ & $\mathrm{I} .08(0.94-\mathrm{I} .24)$ \\
\hline Streptococcal antigen test & $1.21(1.14-1.28)$ & I.II (I.05-I.I8) \\
\hline CRP measurement & $1.51(1.38-1.64)$ & $1.10(1.00-1.21)$ \\
\hline
\end{tabular}

Notes: ${ }^{2}$ Adjusted for sex, date of birth of the child, maternal age, parity, cohabitation status, income, education, smoking status, diagnosis of substance abuse, severe psychiatric disorder, depression and epilepsy as well as use of antiepileptic drugs, antidepressants, benzodiazepines and insulin.

Abbreviations: AP, antipsychotic; $\mathrm{Cl}$, confidence interval; $\mathrm{CRP}, \mathrm{C}$-reactive protein; $\mathrm{GP}$, general practitioner; IRR, incidence rate ratio.
GP contacts could be a proxy for the health status, because the patients must contact the GP for medical advice relating to new symptoms. The GPs act as gatekeepers to specialized health care and complete most of the consultations without referral. ${ }^{15}$ However, severe disease may not be well captured because acute referrals to the emergency department are not included and because GP contacts prompting longer hospitalization and potential follow-up at the hospital might count as only one contact.

We are not aware of other studies investigating the association between prenatal exposure to AP medication and the use of primary health care system in childhood. A recent review by Tosato et $\mathrm{a}^{25}$ concluded that second-generation AP medication is not associated with congenital malformations or short- or long-term child neurodevelopment. In concordance with this, we only found an association between prenatally first-generation AP exposure and contacts to GP, but no associations with second-generation AP medication. 
Table 3 IRR $(95 \% \mathrm{Cl})$ for type of GP contact comparing prenatally AP-exposed children to AP-unexposed children stratified on maternal diagnosis of severe psychiatric disorder

\begin{tabular}{|c|c|c|c|}
\hline & Contacts & Crude IRR (95\% Cl) & Adjusted IRR $(95 \% \mathrm{Cl})^{\mathrm{a}}$ \\
\hline Maternal diagnosis of severe & Total contacts & $1.08(1.01-1.16)$ & $1.03(0.96-1.11)$ \\
\hline \multirow[t]{3}{*}{ psychiatric disorder ${ }^{b}$} & Visits & $1.06(0.99-1.13)$ & $1.02(0.95-1.09)$ \\
\hline & Phone contacts & $1.11(1.02-1.21)$ & $1.05(0.96-1.15)$ \\
\hline & Email contacts (from 2004) & $0.63(0.44-0.91)$ & $0.5 I(0.34-0.77)$ \\
\hline No maternal diagnosis of severe & Total contacts & $1.32(1.27-1.37)$ & $1.08(1.04-1.13)$ \\
\hline \multirow[t]{3}{*}{ psychiatric disorder ${ }^{b}$} & Visits & $1.28(1.23-1.32)$ & $1.08(1.04-1.11)$ \\
\hline & Phone contacts & $1.38(1.32-1.44)$ & $1.09(1.04-1.15)$ \\
\hline & Email contacts (from 2004) & $0.86(0.69-1.09)$ & $0.93(0.74-1.16)$ \\
\hline
\end{tabular}

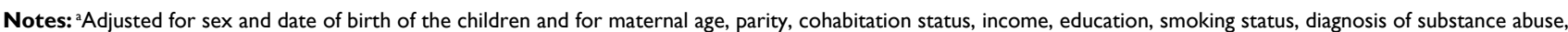
depression and epilepsy as well as use of antiepileptic drugs, antidepressants, benzodiazepines and insulin. ${ }^{b}$ Ever vs never maternal diagnosis of severe psychiatric disorder. Abbreviations: AP, antipsychotic; $\mathrm{Cl}$, confidence interval; GP, general practitioner; IRR, incidence rate ratio.

Table 4 IRR $(95 \% \mathrm{Cl})$ for type of GP contact comparing prenatally AP-exposed children to children of mothers who used AP medication before, but not during pregnancy

\begin{tabular}{lll}
\hline Contacts & $\begin{array}{l}\text { Crude IRR } \\
(\mathbf{9 5 \%} \mathbf{~ C l )}\end{array}$ & $\begin{array}{l}\text { Adjusted IRR } \\
\mathbf{( 9 5 \% ~ C I ) ~}^{\mathbf{a}}\end{array}$ \\
\hline Total contacts & $0.96(0.92-1.00)$ & $0.95(0.9 I-0.99)$ \\
$\quad$ Visits & $0.96(0.93-1.00)$ & $0.96(0.92-1.00)$ \\
Phone contacts & $0.96(0.91-1.00)$ & $0.94(0.89-0.99)$ \\
Email contacts (from 2004) & $0.86(0.67-1.10)$ & $0.76(0.59-0.98)$ \\
\hline
\end{tabular}

Notes: ${ }^{2}$ Adjusted for sex and date of birth of the children and for maternal age, parity, cohabitation status, income, education, smoking status, diagnosis of substance abuse, severe psychiatric disorder, depression and epilepsy as well as use of antiepileptic drugs, antidepressants, benzodiazepines and insulin.

Abbreviations: AP, antipsychotic; $\mathrm{Cl}$, confidence interval; GP, general practitioner; $\mathrm{IRR}$, incidence rate ratio.

\section{Strengths and limitations}

The strengths of the study include the large study population consisting of all live-born singletons in Denmark in the period from 1997 to 2012 with virtually complete follow-up and the use of nationwide registers for collection of data on AP exposure, potential confounders and GP contacts and services. Therefore, selection bias and loss to follow-up are unlikely to have affected our results. The unique personal identification number and the Danish registers with data on all contacts to the health care system, including redemption of prescriptions, and sociodemographic information provided us with relatively accurate and complete data. However, we cannot rule out random misclassification of AP exposure as it is uncertain if all prescribed and redeemed drugs were actually taken, but when we defined the exposed group as women who had two or more redemptions of AP medication during pregnancy, we found almost identical results. Further, a study among pregnant Danish women found high compliance with other psychoactive drugs, such as antiepileptic and antidepressant drugs, but we cannot be certain that this is also

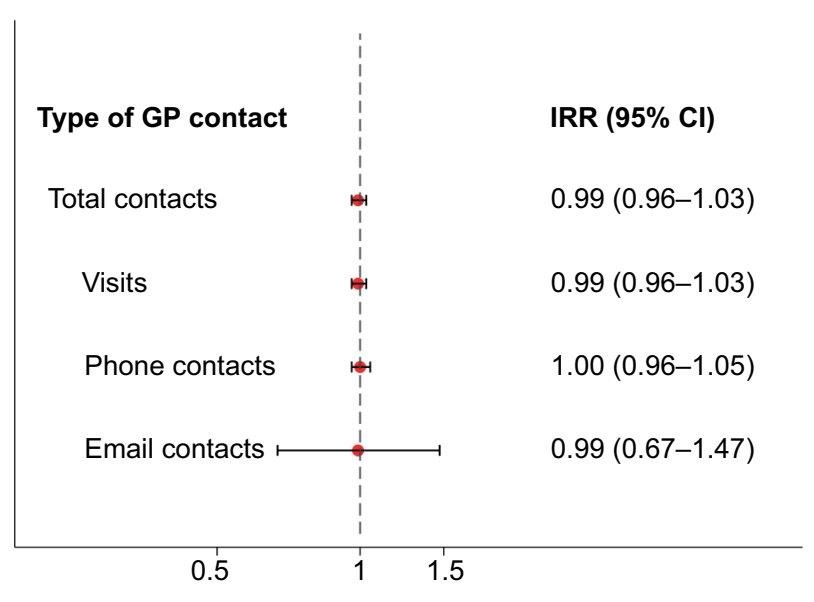

Figure 2 IRR $(95 \% \mathrm{Cl})$ for type of GP contact comparing children prenatally exposed to AP medication with their unexposed siblings.

Notes: IRRs and $95 \% \mathrm{Cls}$ were estimated using a mixed-model analysis with separate strata for each mother and adjusted for sex and date of birth of the children and for maternal age, parity, cohabitation status, income, education, smoking status, diagnosis of substance abuse, depression and epilepsy as well as use of antiepileptic drugs, antidepressants, benzodiazepines and insulin.

Abbreviations: AP, antipsychotic; $\mathrm{Cl}$, confidence interval; GP, general practitioner; IRR, incidence rate ratio.

valid for APs. ${ }^{26}$ The information on the use of GP services was obtained from the Danish National Health Insurance Service Register. The identification of each individual is considered to be accurate as the unique personal identification number is recorded at each GP contact. The completeness of the services registered in the Danish National Health Insurance Service Register is also assumed to be high because the remuneration of the GPs depends on accurate registration of each individual contact and medical procedure. ${ }^{21}$ In addition, the services registered by the GPs are verified by spot checks and algorithms identifying unusual reporting. ${ }^{21}$ The information on exposure and outcome was collected separately and routinely. As this information is not self-reported, the registration of GP contacts has, most likely, not been affected by knowledge of 


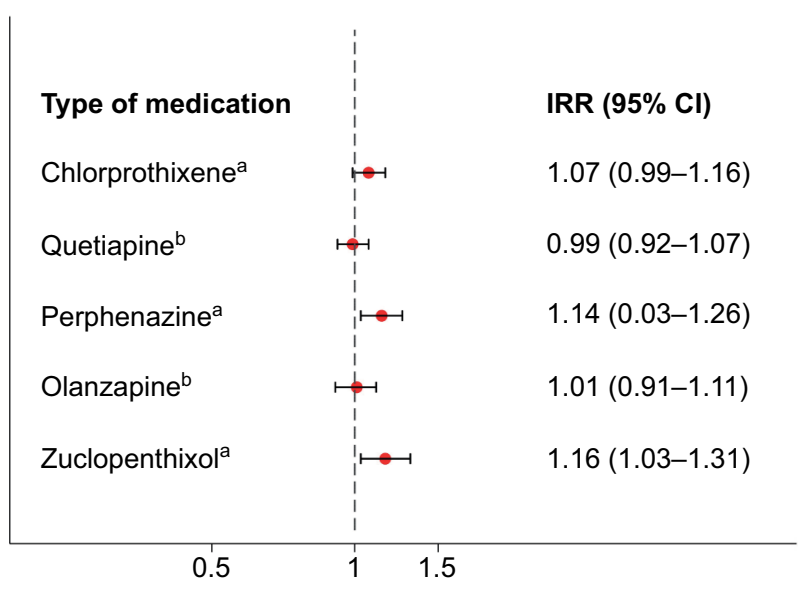

Figure 3 Type of AP medication and IRR $(95 \% \mathrm{Cl})$ for total GP contacts. Notes: IRRs and $95 \% \mathrm{Cls}$ were estimated using negative binomial regression applying cluster robust variance to account for correlations in the children's use of GP services due to multiparity in the mothers. Adjusted for sex and date of birth of the children and for maternal age, parity, cohabitation status, income, education, smoking status, diagnosis of substance abuse, depression and epilepsy as well as use of antiepileptic drugs, antidepressants, benzodiazepines and insulin. ${ }^{2}$ First-generation AP medication. 'Second-generation AP medication.

Abbreviations: AP, antipsychotic; $\mathrm{Cl}$, confidence interval; GP, general practitioner; IRR, incidence rate ratio.

redeemed prescriptions. Still, the potential random misclassification of AP exposure could have caused underestimation of the investigated associations. Adjustment for potential confounders in the main analyses generally attenuated the estimates, which may indicate confounding from these risk factors. In the sensitivity analyses focusing on children born to mothers with severe mental disorder or exclusively on children of mothers who were ever AP medication users, we observed less influence of other potential confounding factors. This could indicate substantial confounding from maternal illness and care-seeking behavior. Unfortunately, we were not able to include information on the severity of the conditions that prompted AP medication treatment. Moreover, confounding from unknown and unmeasured risk factors cannot be excluded.

\section{Alternative explanations of findings}

In the stratified analyses among mothers with a diagnosis of severe psychiatric disorder, we found that the association between prenatal exposure to AP medication and the child's number of GP contacts was weak compared to the main analysis and revealing little difference between crude and adjusted estimates. This is possibly because some of the potential confounding from maternal severe psychiatric disorder was removed. Among mothers with no diagnosis of severe psychiatric disorder, stronger associations between prenatal exposure to AP medication and GP contacts were observed, but substantial confounding was also observed (differences between crude and adjusted estimates). This suggests that we cannot adjust completely for other indications for AP use (e.g., depression, anxiety and other psychiatric disorders) or for other unmeasured risk factors. Furthermore, some women with severe mental disorders are treated exclusively by their GP or by a private practicing psychiatrist; these women have thus not been hospitalized for this condition and are, therefore, not registered with a diagnosis of severe mental disorder in the Danish National Patient Register or the Danish Psychiatric Central Research Register.

We found prenatally AP-exposed children to have slightly fewer GP contacts than children of mothers who used AP medication at some point before, but not during, the index pregnancy. In this sensitivity analysis, we expected to see less confounding by indication from maternal psychiatric disorder. The observed inverse association could indicate that women who stop taking AP medication in the year before a pregnancy might have other characteristics, which could impact the child negatively. A previous study found strong associations between maternal schizophrenia during pregnancy and problems relating to practical baby care, emotional responses and risk of harm to the child as compared to maternal depression. ${ }^{27}$ The sibling analysis showed no differences in the number of GP contacts among children prenatally exposed to AP medication compared to their unexposed siblings. This could indicate that the environment is probably more important than the medication itself, suggesting confounding from environmental factors such as maternal lifestyle and care-seeking behavior. In this analysis, we aimed to adjust for the behavioral effect of the mother. If she had a psychiatric disorder herself and/or took medication, she could be anxious and more likely to contact the GP on behalf of her children. On the other hand, some mothers with severe psychiatric illness might rarely contact the GP on behalf of their children, even not when needed. We assumed that the sibling analysis could remove some of this behavioral effect in the mother because AP-exposed children were only compared to AP-unexposed children born to the same mother. This, however, does not necessarily take into account the potential effect of the severity of maternal disease; mothers with more severe illness may be more likely to have only one child and hence were not included in our analyses.

\section{Conclusion}

In conclusion, we found a difference of $0.32 \mathrm{GP}$ visits per year between prenatally AP-exposed and unexposed children, 
but this is more likely due to confounding from maternal mental illness and behavior than to prenatal exposure to AP medication. When comparing siblings discordant for AP exposure, thereby seeking to control for maternal behavior, we found no difference in the number of GP contacts. This is reassuring considering the potential impact of AP medication on the fetus. This finding also illustrates the complex interaction between indications for AP use, the severity of maternal mental illness and maternal behavior in addition to the inherited genetic and environmental factors that may also affect the child. Our findings could be explained by this multifaceted interplay. Nevertheless, we cannot rule out that a small number of children have frequent GP contact because of increased morbidity due to prenatal exposure to AP medication. AP use during pregnancy requires careful attention to the severity of maternal illness and the maternal behavior, while the risk of potential harm to the fetus and the development of the child should also be considered.

\section{Acknowledgments}

This work was supported by the Program for Clinical Research Infrastructure (PROCRIN) established by the Lundbeck Foundation and the Novo Nordisk Foundation and administered by the Danish Regions.

\section{Author contributions}

BHB and MV initiated the study and obtained the funding. All the authors participated in the design of the study. CHV performed the statistical analyses under the guidance of $\mathrm{BHB}$ and AMLW. AMLW wrote the manuscript. All the authors were involved in the interpretation of data and critical revision of the manuscript. AMLW had the primary responsibility for the final content. All the authors read and approved the final manuscript.

\section{Disclosure}

JC has received honoraria for serving on the Scientific Advisory Board of UCB Nordic and Eisai AB. JC has also received honoraria for giving lectures from UCB Nordic and Eisai AB and has received funding for a trip from UCB Nordic. JC is involved in clinical trials involving the following companies: Pfizer, Inc., Novartis International AG, Eisai AB and Sage Therapeutics, Inc. The other authors report no conflicts of interest in this work.

\section{References}

1. Olfson M, Blanco C, Liu SM, Wang S, Correll CU. National trends in the office-based treatment of children, adolescents, and adults with antipsychotics. Arch Gen Psychiatry. 2012;69(12):1247-1256.
2. Alexander GC, Gallagher SA, Mascola A, Moloney RM, Stafford RS Increasing off-label use of antipsychotic medications in the United States, 1995-2008. Pharmacoepidemiol Drug Saf. 2011;20(2):177-184.

3. Sohn M, Moga DC, Blumenschein K, Talbert J. National trends in offlabel use of atypical antipsychotics in children and adolescents in the United States. Medicine (Baltimore). 2016;95(23):e3784.

4. Epstein RA, Bobo WV, Shelton RC, et al. Increasing use of atypical antipsychotics and anticonvulsants during pregnancy. Pharmacoepidemiol Drug Saf. 2013;22(7):794-801.

5. Toh S, Li Q, Cheetham TC, et al. Prevalence and trends in the use of antipsychotic medications during pregnancy in the U.S., 2001-2007: a population-based study of 585,615 deliveries. Arch Womens Ment Health. 2013;16(2):149-157.

6. Sorensen MJ, Kjaersgaard MI, Pedersen HS, et al. Risk of fetal death after treatment with antipsychotic medications during pregnancy. PLoS One. 2015;10(7): 0132280.

7. Iqbal MM, Aneja A, Rahman A, et al. The potential risks of commonly prescribed antipsychotics: during pregnancy and lactation. Psychiatry (Edgmont). 2005;2(8):36-44.

8. Newport DJ, Calamaras MR, DeVane CL, et al. Atypical antipsychotic administration during late pregnancy: placental passage and obstetrical outcomes. Am J Psychiatry. 2007;164(8):1214-1220.

9. Terrana N, Koren G, Pivovarov J, Etwel F, Nulman I. Pregnancy outcomes following in utero exposure to second-generation antipsychotics: a systematic review and meta-analysis. J Clin Psychopharmacol. 2015;35(5):559-565.

10. Coughlin CG, Blackwell KA, Bartley C, Hay M, Yonkers KA, Bloch $\mathrm{MH}$. Obstetric and neonatal outcomes after antipsychotic medication exposure in pregnancy. Obstet Gynecol. 2015;125(5):1224-1235.

11. Kulkarni J, Storch A, Baraniuk A, Gilbert H, Gavrilidis E, Worsley R. Antipsychotic use in pregnancy. Expert Opin Pharmacother. 2015;16(9):1335-1345.

12. Peng M, Gao K, Ding Y, et al. Effects of prenatal exposure to atypical antipsychotics on postnatal development and growth of infants: a case-controlled, prospective study. Psychopharmacology (Berl). 2013;228(4):577-584.

13. Johnson KC, LaPrairie JL, Brennan PA, Stowe ZN, Newport DJ. Prenatal antipsychotic exposure and neuromotor performance during infancy. Arch Gen Psychiatry. 2012;69(8):787-794.

14. Pedersen KM, Andersen JS, Sondergaard J. General practice and primary health care in Denmark. J Am Board Fam Med. 2012;25(suppl 1): S34-S38.

15. Christiansen T. Organization and financing of the Danish health care system. Health Policy. 2002;59(2):107-118.

16. Wurtz AM, Rytter D, Vestergaard CH, Christensen J, Vestergaard M, Bech BH. Prenatal exposure to antiepileptic drugs and use of primary healthcare during childhood: a population-based cohort study in Denmark. BMJ Open. 2017;7(1):e012836.

17. Schmidt M, Schmidt SA, Sandegaard JL, Ehrenstein V, Pedersen L, Sorensen HT. The Danish National Patient Registry: a review of content, data quality, and research potential. Clin Epidemiol. 2015;7:449-490.

18. Pedersen CB. The Danish Civil Registration System. Scand J Public Health. 2011;39(7 suppl):22-25.

19. Kildemoes HW, Sorensen HT, Hallas J. The Danish National Prescription Registry. Scand J Public Health. 2011;39(7 suppl):38-41.

20. WHO Collaborating Centre for Drug Statistics Methodology (WHOCC) [webpage on the Internet]. Anatomical Therapeutic Chemical (ATC) Classification System. Oslo, Norway: 2011. Available from: http://www. whocc.no/atc/structure_and_principles/. Accessed October 14, 2017.

21. Andersen JS, Olivarius Nde F, Krasnik A. The Danish National Health Service Register. Scand J Public Health. 2011;39(7 suppl):34-37.

22. Statistics Denmark [database on the Internet]. Statistics Denmark. Denmark. Available from: http://www.dst.dk/en. Accessed October 14, 2017.

23. Mors O, Perto GP, Mortensen PB. The Danish Psychiatric Central Research Register. Scand J Public Health. 2011;39(7 suppl):54-57. 
24. Lynge E, Sandegaard JL, Rebolj M. The Danish National Patient Register. Scand J Public Health. 2011;39(7 suppl):30-33.

25. Tosato $\mathrm{S}$, Albert $\mathrm{U}$, Tomassi $\mathrm{S}$, et al. A systematized review of atypical antipsychotics in pregnant women: balancing between risks of untreated illness and risks of drug-related adverse effects. J Clin Psychiatry. 2017;78(5):e477-e489.
26. Olesen C, Sondergaard C, Thrane N, et al. Do pregnant women report use of dispensed medications? Epidemiology. 2001;12(5):497-501.

27. Howard LM, Thornicroft G, Salmon M, Appleby L. Predictors of parenting outcome in women with psychotic disorders discharged from mother and baby units. Acta Psychiatr Scand. 2004;110(5):347-355.

\section{Publish your work in this journal}

Clinical Epidemiology is an international, peer-reviewed, open access, online journal focusing on disease and drug epidemiology, identification of risk factors and screening procedures to develop optimal preventative initiatives and programs. Specific topics include: diagnosis, prognosis, treatment, screening, prevention, risk factor modification,

Submit your manuscript here: https://www.dovepress.com/clinical-epidemiology-journa
Dovepress

systematic reviews, risk and safety of medical interventions, epidemiology and biostatistical methods, and evaluation of guidelines, translational medicine, health policies and economic evaluations. The manuscript management system is completely online and includes a very quick and fair peer-review system, which is all easy to use. 